\title{
Effective medium approach for heterogeneous reaction-diffusion media
}

\author{
Sergio Alonso, ${ }^{1, a)}$ Markus Bär, ${ }^{1}$ and Raymond Kapral ${ }^{2}$ \\ ${ }^{1}$ Physikalisch-Technische Bundesanstalt, 10587 Berlin, Germany \\ ${ }^{2}$ Department of Chemistry, University of Toronto, Toronto, Ontario M5S 3H6, Canada
}

(Received 28 September 2009; accepted 27 October 2009; published online 1 December 2009)

\begin{abstract}
An effective medium theory that can be used to calculate effective diffusion and reaction rate coefficients in random heterogeneous reaction-diffusion systems is described. The predictions of the theory are compared with simulations of spatially distributed media with different types of heterogeneity. The magnitude of the front velocity in bistable media is used to gauge the accuracy of the theoretical predictions. Quantitative agreement is found if the diffusion length in the heterogeneities is large compared to the characteristic width of the front. However, for small diffusion lengths the agreement depends on the type of heterogeneity. The effective medium predictions are also compared with simulations on systems with regular or temporal disorder.
\end{abstract}

(C) 2009 American Institute of Physics. [doi:10.1063/1.3265987]

\section{INTRODUCTION}

Reaction-diffusion systems maintained far from equilibrium often possess spatio-temporal structure. ${ }^{1}$ Experimental and analytical studies show that a large variety of patterns can exist, for example, traveling waves and spiral waves, due to the interplay between diffusion and nonlinear reaction kinetics. ${ }^{2}$ Pattern formation has been observed in extended chemical media ${ }^{3}$ and plays a central role in the dynamics of many biological systems. ${ }^{4}$ The models describing such processes often assume that the system is spatially homogeneous and provide a reasonable description of many systems. There is, however, an increasing interest in physical systems where heterogeneity is important and models for such systems involve heterogeneous reaction-diffusion equations where the diffusion coefficients and reaction rate constants depend explicitly on space.

The Belousov-Zhabotinsky reaction provides an example of a system where external heterogeneities in an extended chemical medium can influence pattern formation. The use of masks and lithographic techniques permits the introduction in the reaction of patterned illumination ${ }^{5}$ and patterned distribution of catalyst, ${ }^{6}$ respectively. Both methods can be used to produce inhomogeneities. Another example of heterogeneous chemical pattern formation is the interaction between waves and nonreactive $T i$ inclusions in the catalytic oxidation of $\mathrm{CO}$ on Pt surfaces. ${ }^{7}$ In these examples the heterogeneities are large compared with the characteristic size of the pattern formed in system. Heterogeneities have been introduced by confining chemical species in small compartments. Examples include reactions in microemulsion systems ${ }^{8}$ and on porous catalytic beads. ${ }^{9,10}$ In the microemulsion experiments the characteristic sizes of the heterogeneities and the pattern type can be controlled by varying the composition of the system. ${ }^{11}$

Heterogeneities are an intrinsic feature in biological reaction-diffusion systems. Electrochemical waves propagate

${ }^{\text {a)} E l e c t r o n i c ~ m a i l: ~ s e r g i o . a l o n s o @ p t b . d e . ~}$ in cardiac tissue and are responsible for the pumping action of the heart. Cardiac tissue is highly inhomogeneous and the effect of heterogeneities, such as dead cells ${ }^{12,13}$ or randomly distributed connections among the cells, ${ }^{14}$ have been studied numerically with heterogeneous reaction-diffusion cardiac models. These studies show that the propagation velocity depends mainly on the fraction of heterogeneities in the tissue. Experimental and numerical studies on electrochemical propagation through cardiac cell cultures, that are typically more inhomogeneous than the tissue, show similar properties. ${ }^{15,16}$ Heterogeneous models have been employed to study calcium waves in cells ${ }^{17,18}$ and the interactions among neurons. ${ }^{19}$

There have been several previous investigations of homogenization of particular heterogeneous reaction-diffusion systems by analytical ${ }^{20,21}$ or numerical averaging ${ }^{22}$ methods. The results of these studies depend on the particular features of the model. Here, we describe a general method, which does not depend on the details of the model, to obtain effective reaction rates and diffusion coefficients for heterogeneous reaction-diffusion systems with an arbitrary number of chemical species and possibly nonlinear kinetics. ${ }^{23}$ However, we restrict our numerical simulations to binary media with two phases, 1 and 2, with fractions $1-\phi$ and $\phi$, respectively. Earlier studies considered the case of linear reactions. ${ }^{24} \mathrm{We}$ present analytical expressions for the effective medium properties and validate the results by comparisons with simulations of the velocity of fronts in a bistable heterogeneous reaction-diffusion system.

The manuscript is organized as follows. In the next section, we give expressions for the effective reaction rates and diffusion coefficients in terms of the fractions of heterogeneities for a set of reaction-diffusion equations. The various kinds of heterogeneity under study here are specified in Sec. III, while Sec. IV is devoted to the description of the numerical simulations for a single component reaction-diffusion system, where the specification of the different types of possible heterogeneity is crucial. In Sec. V we present a discussion of further applications of the effective medium theory. 


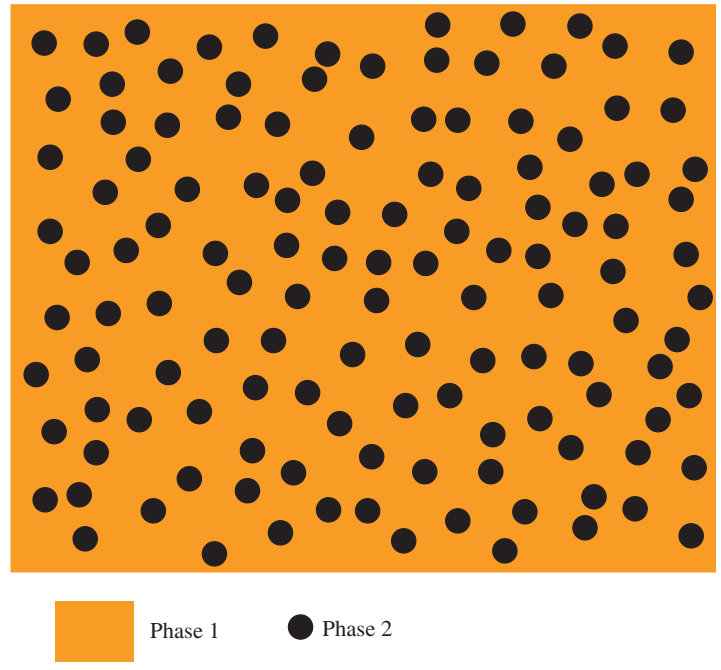

FIG. 1. Sketch of a random distribution of phase 2 in a background phase 1 . Here, the size of the heterogeneities (phase 2) determines characteristic scale $\ell$.

We show cases where numerical and theoretical results agree and also discuss the limitations of the theory. The final section presents conclusions and further discussion of the results.

\section{EFFECTIVE MEDIUM REACTION-DIFFUSION EQUATIONS}

Consider a system where domains of phase 2 are dispersed in a medium of phase 1 (see Fig. 1). Chemical species labeled $\alpha=1, \ldots, n$ undergo reaction and diffusion dynamics in the system. Particles of species $\alpha$ can diffuse throughout the system but their diffusion coefficients may differ in the two phases.

We assume that reaction can occur in domains of both phases. If the domains are taken to be sufficiently large that a continuum description is applicable, the dynamics in the heterogeneous medium is given by the reaction-diffusion equation, ${ }^{25}$

$$
\frac{\partial c_{\alpha}(\mathbf{r}, t)}{\partial t}=\nabla \cdot\left(D_{\alpha}(\mathbf{r}) \nabla c_{\alpha}(\mathbf{r}, t)\right)+\mathcal{R}_{\alpha}(\mathbf{c}(\mathbf{r}, t)) .
$$

Spatially dependent diffusion coefficients are defined so that $D_{\alpha}(\mathbf{r})=D_{\alpha}^{(i)}$ in phase $i=1,2$. If there are $N$ domains of phase 2 then the reaction term takes the form

$$
\begin{aligned}
\mathcal{R}_{\alpha}(\mathbf{c}(\mathbf{r}, t))= & \left(\sum_{k=1}^{N} R_{\alpha}^{(2)}(\mathbf{c}(\mathbf{r}, t)) \theta_{k}(\mathbf{r})\right) \\
& +R_{\alpha}^{(1)}(\mathbf{c}(\mathbf{r}, t))\left(1-\theta_{k}(\mathbf{r})\right),
\end{aligned}
$$

where $\theta_{k}(\mathbf{r})$ is unity in the $k$ th domain of phase 2 and zero otherwise. The domains are assumed to have a characteristic size $\ell$ and the volume fraction of phase 2 in the system is $\phi$.

If the characteristic domain size in this heterogeneous system is small compared to the scales of interest, one may describe the reaction-diffusion dynamics in terms of effective medium equations obtained by coarse graining over distances that are large compared to the sizes of the inclusions of phase 2 in phase 1 . Letting the angular bracket $\langle\cdots\rangle$ represent such a coarse-graining average, we are interested in the reduction of the average of Eq. (1),

$$
\frac{\partial\left\langle c_{\alpha}(\mathbf{r}, t)\right\rangle}{\partial t}=\left\langle\nabla \cdot\left(D_{\alpha}(\mathbf{r}) \nabla c_{\alpha}(\mathbf{r}, t)\right)\right\rangle+\left\langle\mathcal{R}_{\alpha}(\mathbf{c}(\mathbf{r}, t))\right\rangle,
$$

to an effective medium reaction-diffusion equation of the form

$$
\frac{\partial\left\langle c_{\alpha}(\mathbf{r}, t)\right\rangle}{\partial t}=D_{\alpha}^{e} \nabla^{2}\left\langle c_{\alpha}(\mathbf{r}, t)\right\rangle+R_{\alpha}^{e}(\langle\mathbf{c}(\mathbf{r}, t)\rangle),
$$

characterized by effective diffusion coefficients $D_{\alpha}^{e}$ and effective reaction rates $R_{\alpha}^{e}(\langle\mathbf{c}(\mathbf{r}, t)\rangle)$.

To derive the effective reaction-diffusion equation we use a method developed earlier for linear reaction kinetics ${ }^{24}$ but focus on nonlinear reaction-controlled kinetics in order to study pattern-forming reaction-diffusion media lying far from equilibrium. We begin by introducing fictitious background diffusion coefficients $D_{\alpha}^{m}$ and rewriting the Eq. (1) in terms of these diffusion coefficients as

$$
\left(\frac{\partial}{\partial t}-D_{\alpha}^{m} \nabla^{2}\right) c_{\alpha}(\mathbf{r}, t)=-\nabla \cdot \delta \mathbf{j}_{\alpha}(\mathbf{r}, t)+\mathcal{R}_{\alpha}(\mathbf{c}(\mathbf{r}, t)),
$$

where

$$
\delta \mathbf{j}_{\alpha}(\mathbf{r}, t)=-\delta D_{\alpha}(\mathbf{r}) \nabla c_{\alpha}(\mathbf{r}, t),
$$

and we have defined $\delta D_{\alpha}(\mathbf{r})=D_{\alpha}(\mathbf{r})-D_{\alpha}^{m}$. In Fourier space $\left(\hat{h}(\omega)=\int d t e^{i \omega t} h(t)\right)$ this equation takes the form

$$
\left(-i \omega-D_{\alpha}^{m} \nabla^{2}\right) \hat{c}_{\alpha}(\mathbf{r}, \omega)=-\nabla \cdot \delta \hat{\mathbf{j}}_{\alpha}(\mathbf{r}, \omega)+\hat{\mathcal{R}}_{\alpha}(\mathbf{r}, \omega),
$$

whose solution is

$$
\begin{aligned}
\hat{c}_{\alpha}(\mathbf{r}, \omega)= & -\int d \mathbf{r}^{\prime} G_{\alpha}^{m}\left(\mathbf{r}-\mathbf{r}^{\prime}, \omega\right) \nabla^{\prime} \cdot \delta \hat{\mathbf{j}}_{\alpha}\left(\mathbf{r}^{\prime}, \omega\right) \\
& +\int d \mathbf{r}^{\prime} G_{\alpha}^{m}\left(\mathbf{r}-\mathbf{r}^{\prime}, \omega\right) \hat{\mathcal{R}}_{\alpha}\left(\mathbf{r}^{\prime}, \omega\right) .
\end{aligned}
$$

The diffusion Green function depends on the spatial dimension, and it is

$$
G_{\alpha}^{m}(\mathbf{r}, \omega)=\frac{-e^{-\sigma(\omega) r}}{2 D_{\alpha}^{m} \sigma(\omega)},
$$

in $d=1$,

$$
G_{\alpha}^{m}(\mathbf{r}, \omega)=\frac{i}{4 D_{\alpha}^{m}} H_{o}^{(1)}\left(\frac{\sigma(\omega)}{i} r\right),
$$

in $d=2$, where $H_{o}^{(1)}$ is a Hankel function, and

$$
G_{\alpha}^{m}(\mathbf{r}, \omega)=\frac{e^{-\sigma(\omega) r}}{4 \pi D_{\alpha}^{m} r},
$$

in $d=3$. In the above expressions we have used the parameter: $\sigma(\omega)=\left(-i \omega / D_{\alpha}^{m}\right)^{1 / 2}$.

\section{A. Reaction}

We assume that phase 2 is randomly dispersed in phase 1. Given that the characteristic size of phase 2 domains is $\ell$, 
we divide the system into cells with this size so that each cell contains either phase 1 or phase 2 . We may now distinguish contributions coming from the same cell (self-contributions) and from different cells (local contributions). We make this distinction because, while the dynamics is strongly correlated within a domain, correlations among domains are weak on the coarse graining scale. The self-contributions arising from the same cell $\left(c_{\alpha}(\mathbf{r}, \omega)\right)_{s}$ may be obtained by taking $\mathbf{r}^{\prime}$ to lie in the same cell as $\mathbf{r}$ in Eq. (8) to obtain

$$
\left(c_{\alpha}(\mathbf{r}, \omega)\right)_{s}=\left[\int_{\left|\mathbf{r}-\mathbf{r}^{\prime}\right|<\ell} d \mathbf{r}^{\prime} G_{\alpha}^{m}\left(\mathbf{r}-\mathbf{r}^{\prime}, \omega=0\right)\right] \hat{\mathcal{R}}_{\alpha}(\mathbf{r}, \omega) .
$$

Within a domain, diffusion provides rapid mixing so that the Green function can be replaced by its zero frequency value and the reaction rate can be replaced by its value at the cell center. When Eq. (8) is integrated over the domain volume, the first term on the right hand side vanishes. If we consider a spherical volume with radius $\ell$ centered at $\mathbf{r}$ to evaluate the integral over the Green function, we obtain

$$
g_{s} \equiv \int_{\left|\mathbf{r}-\mathbf{r}^{\prime}\right|<\ell} d \mathbf{r}^{\prime} G_{\alpha}^{m}\left(\mathbf{r}-\mathbf{r}^{\prime}, \omega=0\right)=\frac{\ell^{2}}{2 D_{\alpha}^{m}} \equiv \tau_{D},
$$

which defines the diffusion time $\tau_{D}$. Equivalent results are obtained in one-dimensional (1D) and two-dimensional (2D) systems using expressions (9) and (10), respectively. Consequently the self-contribution to the concentration field is

$$
\left(c_{\alpha}(\mathbf{r}, \omega)\right)_{s}=\tau_{D} \hat{\mathcal{R}}_{\alpha}(\mathbf{r}, \omega) .
$$

Since the reaction rate scales as $\tau_{R}^{-1}$, the reciprocal of the characteristic reaction time, the self-contribution scales as $\left(c_{\alpha}(\mathbf{r}, \omega)\right)_{s} \sim \tau_{D} / \tau_{R}$. For reaction controlled kinetics we have $\tau_{D} / \tau_{R} \ll 1$ and the self-contribution is small. The local concentration field defined as $\left(c_{\alpha}(\mathbf{r}, \omega)\right)_{l}=c_{\alpha}(\mathbf{r}, \omega)-\left(c_{\alpha}(\mathbf{r}, \omega)\right)_{s}$ will then provide a good approximation to the full concentration field since $\left(c_{\alpha}(\mathbf{r}, \omega)\right)_{l}=c_{\alpha}(\mathbf{r}, \omega)+\mathcal{O}\left(\tau_{D} / \tau_{R}\right)$. In this reaction controlled limit we may replace $c_{\alpha}(\mathbf{r}, t)$ by $c_{\alpha, l}(\mathbf{r}, t)$ in the reaction rate, $\mathcal{R}_{\alpha}(\mathbf{c}(\mathbf{r}, t)) \approx \mathcal{R}_{\alpha}\left(\mathbf{c}_{l}(\mathbf{r}, t)\right)$. The effective reaction rate is then given by $\left\langle\mathcal{R}_{\alpha}\left(\mathbf{c}_{l}(\mathbf{r}, t)\right)\right\rangle$. Taking into account the definition of $\mathcal{R}_{\alpha}(\mathbf{c}(\mathbf{r}, t))$ in Eq. (2) we may write

$$
\begin{aligned}
\left\langle\mathcal{R}_{\alpha}(\mathbf{c}(\mathbf{r}, t))\right\rangle \approx R_{\alpha}^{e}= & (1-\phi) R_{\alpha}^{(1)}(\langle\mathbf{c}(\mathbf{r}, t)\rangle) \\
& +\phi R_{\alpha}^{(2)}(\langle\mathbf{c}(\mathbf{r}, t)\rangle) .
\end{aligned}
$$

In writing this equation we have used the fact that reaction terms depending on the local concentrations are uncorrelated and have represented the (nonlinear) reaction rate in a local mean field approximation within the coarse grained cells terms of the averaged local concentration fields. This local mean field approximation will retain correlations arising on long distance scales needed to describe nonequilibrium pattern formation.

For linear reaction rates the condition $\tau_{D} / \tau_{R} \ll 1$ can be relaxed and the averages can be done ${ }^{24}$ giving rise to a more complete expression for $R_{\alpha}^{e}$. However, the average of nonlin- ear reaction rates is more complicated and to obtain a tractable expression we have restricted ourselves to systems where this condition is satisfied.

\section{B. Diffusion}

Next we turn to the evaluation of the effective diffusion coefficients. The derivation parallels that in Ref. 24 but extended to $d$ dimensions. The effective diffusion coefficients are defined from the expression for the average of the diffusion flux in terms of the average concentration gradient,

$$
\left\langle\delta \hat{\mathbf{j}}_{\alpha}(\mathbf{r}, \omega)\right\rangle=-\delta D_{\alpha}^{e}\left\langle\nabla c_{\alpha}(\mathbf{r}, \omega)\right\rangle .
$$

The self-contribution may be computed by taking the gradient of Eq. (8) and integrating within a cell to obtain,

$$
\left(\nabla c_{\alpha}\right)_{s}(\mathbf{r}, \omega)=g_{d} \delta \hat{\mathbf{j}}_{\alpha}(\mathbf{r}, \omega),
$$

where

$$
g_{d} 1=-\left[\int_{\left|\mathbf{r}-\mathbf{r}^{\prime}\right|<\ell} d \mathbf{r}^{\prime} \nabla \nabla G_{\alpha}^{m}\left(\mathbf{r}-\mathbf{r}^{\prime}, \omega\right)\right]=\frac{1}{d D_{\alpha}^{m}} 1,
$$

$d$ is the spatial dimension and 1 is a unit tensor. The evaluation of the integral in Eq. (18) was carried out in $d$ dimensions using the appropriate $d$-dimensional Green functions. The local contribution is defined to be

$$
\begin{aligned}
\left(\nabla c_{\alpha}\right)_{l}(\mathbf{r}, \omega) & =\nabla c_{\alpha}(\mathbf{r}, \omega)-\left(\nabla c_{\alpha}\right)_{s}(\mathbf{r}, \omega) \\
& =\left(1+g_{d} \delta D_{\alpha}(\mathbf{r})\right) \nabla c_{\alpha}(\mathbf{r}, \omega),
\end{aligned}
$$

or

$$
\nabla c_{\alpha}(\mathbf{r}, \omega)=\left(1+g_{d} \delta D_{\alpha}(\mathbf{r})\right)^{-1}\left(\nabla c_{\alpha}\right)_{l}(\mathbf{r}, \omega) .
$$

We may now obtain an expression for the diffusion flux in terms of the local concentration gradient as

$$
\begin{aligned}
\delta \hat{\mathbf{j}}_{\alpha}(\mathbf{r}, \omega) & =-\delta D_{\alpha}(\mathbf{r}) \nabla c_{\alpha}(\mathbf{r}, \omega) \\
& =-\delta D_{\alpha}(\mathbf{r})\left(1+g_{d} \delta D_{\alpha}(\mathbf{r})\right)^{-1}\left(\nabla c_{\alpha}\right)_{l}(\mathbf{r}, \omega) .
\end{aligned}
$$

Neglecting correlations among the volume elements, the average of this equation yields,

$$
\left\langle\delta \hat{\mathbf{j}}_{\alpha}(\mathbf{r}, \omega)\right\rangle \approx-\left\langle\delta D_{\alpha}(\mathbf{r})\left(1+g_{d} \delta D_{\alpha}(\mathbf{r})\right)^{-1}\right\rangle\left\langle\left(\nabla c_{\alpha}\right)_{l}(\mathbf{r}, \omega)\right\rangle .
$$

To complete the derivation we need an expression for $\left\langle\left(\nabla c_{\alpha}\right)_{l}(\mathbf{r}, \omega)\right\rangle$ in terms of $\left\langle\nabla c_{\alpha}(\mathbf{r}, \omega)\right\rangle$. From Eqs. (17) and (19) we have

$$
\begin{aligned}
\left\langle\left(\nabla c_{\alpha}\right)_{l}(\mathbf{r}, \omega)\right\rangle & =\left\langle\nabla c_{\alpha}(\mathbf{r}, \omega)\right\rangle-\left\langle\left(\nabla c_{\alpha}\right)_{s}(\mathbf{r}, \omega)\right\rangle \\
& =\left\langle\nabla c_{\alpha}(\mathbf{r}, \omega)\right\rangle-g_{d}\left\langle\delta \hat{\mathbf{j}}_{\alpha}(\mathbf{r}, \omega)\right\rangle,
\end{aligned}
$$

and using Eq. (22) we obtain

$$
\left\langle\left(\nabla c_{\alpha}\right)_{l}(\mathbf{r}, \omega)\right\rangle=\frac{\left\langle\nabla c_{\alpha}(\mathbf{r}, \omega)\right\rangle}{1-g_{d}\left\langle\delta D_{\alpha}(\mathbf{r})\left(1+g_{d} \delta D_{\alpha}(\mathbf{r})\right)^{-1}\right\rangle} .
$$

Substitution into Eq. (22) and comparison with Eq. (16) yield an expression for the effective diffusion coefficient, 


$$
\delta D_{\alpha}^{e}=\frac{\left\langle\delta D_{\alpha}(\mathbf{r})\left(1+g_{d} \delta D_{\alpha}(\mathbf{r})\right)^{-1}\right\rangle}{1-g_{d}\left\langle\delta D_{\alpha}(\mathbf{r})\left(1+g_{d} \delta D_{\alpha}(\mathbf{r})\right)^{-1}\right\rangle} .
$$

Using the fact that $D_{\alpha}^{e}=D_{\alpha}^{m}+\delta D_{\alpha}^{e}$ and rearranging, we find

$$
\frac{D_{\alpha}^{e}-D_{\alpha}^{m}}{1+g_{d}\left(D_{\alpha}^{e}-D_{\alpha}^{m}\right)}=\left\langle\delta D_{\alpha}(\mathbf{r})\left(1+g_{d} \delta D_{\alpha}(\mathbf{r})\right)^{-1}\right\rangle .
$$

To determine the effective diffusion coefficients we may choose $D_{\alpha}^{m}=D_{\alpha}^{e}$ so that Eq. (26) for the case of binary media yields

$$
\frac{D_{\alpha}^{(1)}-D_{\alpha}^{e}}{D_{\alpha}^{(1)}+(d-1) D_{\alpha}^{e}}(1-\phi)+\frac{D_{\alpha}^{(2)}-D_{\alpha}^{e}}{D_{\alpha}^{(2)}+(d-1) D_{\alpha}^{e}} \phi=0,
$$

for a $d$-dimensional system. This expression recovers the old formula of Bruggeman ${ }^{26}$ for the calculation of effective variables for the conductivity of mixtures of isotropic materials. Equivalent expression has been obtained for spherical inclusions in a conducting material ${ }^{27}$ and for inhomogeneous transport in resistor networks. ${ }^{28,29}$ Note also that for the case $d=1$, Eq. (27) yields the weighted harmonic mean: $1 / D_{\alpha}^{e}$ $=(1-\phi) / D_{\alpha}^{(1)}+\phi / D_{\alpha}^{(2)}$.

The above expression for the diffusion coefficient predicts a percolation threshold for $D_{\alpha}^{(2)}=0$ of $\phi^{*}=1 / d$ for $d$ $=2,3$ because the effective diffusion coefficient becomes zero at this value of $\phi$, since $\left(D_{\alpha}^{e}=D_{\alpha}^{(1)}(1-d \phi)\right)$. The existence of such threshold is one of the differences between this effective theory and others. ${ }^{27}$

Finally, we note that our analysis of effective diffusion is restricted to the case where the diffusion matrix is diagonal. Cross diffusion effects can play an important role in some heterogeneous media ${ }^{30}$ and an extension of our analysis to treat such situations should be carried out.

\section{MODELS}

We consider a simple model of bistable media described by the following reaction-diffusion equation:

$$
\frac{\partial c}{\partial t}=R(c, r)+\nabla \cdot(D(r) \nabla c),
$$

where $R(c, r)=k(r) c(1-c)(c-a)$ is the reaction rate and $D(r)$ is the diffusion coefficient. Under homogeneous conditions (i.e., without heterogeneities), the values of the parameters are independent of space, $D(r)=D^{(1)}$ and $k(r)=k^{(1)}$, and a front may propagate with a stationary shape and constant velocity,

$$
V_{0}=\sqrt{\frac{D^{(1)} k^{(1)}}{2}}(1-2 a)
$$

A fraction of heterogeneities with different reaction $\left(k^{(2)}\right)$ and diffusion $\left(D^{(2)}\right)$ coefficients, corresponding to phase 2 , is dispersed in normal phase 1 with specified values of the reaction $\left(k^{(1)}=50\right)$ and the diffusion $\left(D^{(1)}=1\right)$ coefficients. In order to study this system, two strategies are considered. First, we use the theoretical approximation introduced above and integrate a continuous medium reaction-diffusion equation with an effective reaction rate and diffusion coefficients. Second, we consider a heterogeneous system with the same frac- tion of phase 2 and assume heterogeneities exist on the scale of the discretization length used in the simulation of the reaction-diffusion system.

\section{A. Effective-medium model}

The dependence of the front velocity on $\phi$ and $D^{(2)}$ of phase 2 can be calculated using the effective values of the reaction rate $k^{e}$ and diffusion $D^{e}$ coefficients obtained in the previous section. Under homogeneous conditions the front velocity can be calculated analytically using Eq. (29) but with the effective values of the transport coefficients,

$$
V=\sqrt{\frac{D^{e} k^{e}}{2}}(1-2 a)
$$

For the binary mix considered here, the effective diffusion coefficient can be obtained from Eq. (27),

$$
D^{e}=\frac{\bar{D}+\sqrt{\bar{D}^{2}+(d-1) D^{(1)} D^{(2)}}}{(d-1)},
$$

where $\bar{D}=\left(D^{(1)}(d(1-\phi)-1)+D^{(2)}(d \phi-1)\right) / 2$. In addition, the dependence of the effective reaction rate on the fraction $\phi$ can be derived from Eq. (15) as

$$
k^{e}=(1-\phi) k \text {. }
$$

The diffusion coefficient in Eq. (31) depends on the spatial dimension of the system $d$; thus, while the velocities of traveling fronts are independent of the spatial dimension, apart from curvature effects, for homogeneous media, this is not the case for heterogeneous media.

\section{B. Models with discrete heterogeneities}

Equation (28) was integrated using the Euler method for the temporal evolution and finite differences for the spatial discretization. The five-point and seven-point formula for the calculation of the Laplacian in 2D and three-dimensional (3D) systems, respectively, were employed. The continuous medium described by Eq. (28) was replaced by a discrete square (2D) or cubic (3D) grid where each site is connected by coupling to the nearest neighbors [Fig. 2(a)].

The flux $(\vec{J}=-D \vec{\nabla} c$ in the continuum description) can be calculated between two nodes of the grid in the discrete description from $J_{i j}=-D_{i j}\left(c_{i}-c_{j}\right) / \Delta x$, where $D_{i j}$ is the arithmetic mean of the diffusion coefficients in nodes $i$ and $j$ : $D_{i j}=\left(D_{i}+D_{j}\right) / 2$. However, this expression can give rise to nonphysical results in extreme cases. For example, when one of the diffusion coefficients is zero, one does not expect any flux between the two grid points, but from the previous expression, if the other diffusion coefficient is nonzero, a flux does exist. To avoid such nonphysical conditions, we consider two interconnected grids, one for the nodes $\left(N_{i}, N_{j}, N_{k}, \ldots\right)$ and other for the links between nodes $\left(L_{i j}, L_{j k}, L_{k l}, \ldots\right)$. In summary, we consider a grid of links, where the transport occurs, coupled to a grid of nodes, where the reaction takes place. Under homogeneous conditions this does not affect the usual description of discrete reactiondiffusion equations. 
homogeneous system

a)

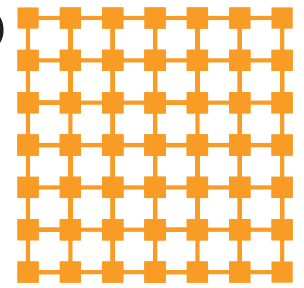

weak links

c)

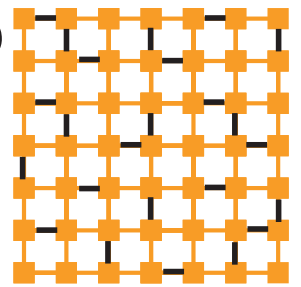

reactive defects

e)

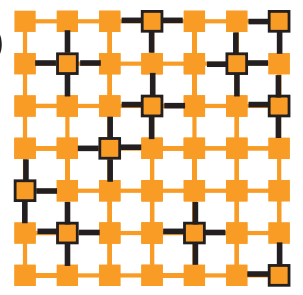

weak nodes

b)

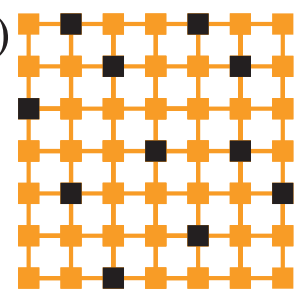

weak links and weak nodes

d)

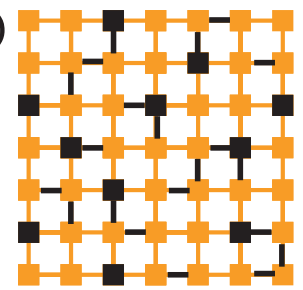

non-reactive defects

f)

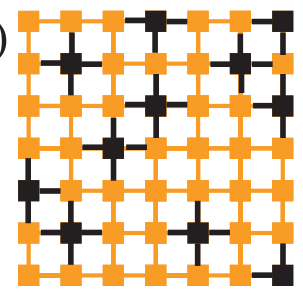

FIG. 2. Sketch of the homogeneous and heterogeneous discrete reactiondiffusion systems. Bright squares and links correspond to normal grid nodes $\left(k^{(1)}\right)$ and normal links $\left(D^{(1)}\right)$, respectively. Black squares and links correspond to nonreactive nodes $\left(k^{(2)}\right)$ and weak links $\left(D^{(2)}\right)$, respectively.

We employ the parameters $k(r)$ and $D(r)$ to introduce the heterogeneities in the numerical simulations. Different scenarios are studied here, and a sketch of each type is shown in Fig. 2.

(1) Weak nodes: First, we suppose that a fraction $\left(\phi_{N}\right)$ of the nodes $\left(N_{i}\right)$ are weakly reactive $\left(k_{i}=k^{(2)}<k^{(1)}\right)$. The diffusion between nodes is, however, completely homogeneous [Fig. 2(b)].

(2) Weak links: We may also consider the case where a link $\left(L_{i j}\right)$ between two neighboring sites $\left(N_{i}, N_{j}\right)$ may be associated with normal diffusion $\left(D^{(1)}\right)$ or has a reduced diffusion coefficient $\left(D^{(2)}\right)$ [Fig. 2(c)]. We introduce a fraction $\phi_{L}$ of these weak links. We do not consider correlations among their positions and assume homogeneous reaction. This structure allows for bond percolation at $\phi=\phi^{*}$ in the limit $D^{(2)}=0$.

(3) Weak links and nodes: Alternatively, we may consider a system with both weak links and weak nodes, assuming that both types of heterogeneities appear with the same probability $\phi_{N}=\phi_{L}$ [Fig. 2(d)]. We do not consider correlations between the locations of the two types of heterogeneities.

(4) Reactive defects: We also consider defects in the system. A defect corresponds to a node $\left(N_{i}\right)$ with a reduced diffusion $\left(D^{(2)}\right)$ in all the associated links $\left(L_{i j}\right)$, see Fig. $2(\mathrm{e})$. The defects appear with probability $\phi_{D}$. It is interesting to note that this type of heterogeneity gives a)
Fronts in 2D
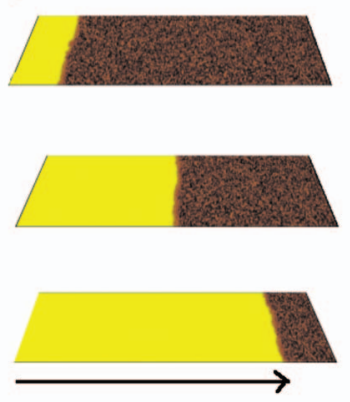

$L^{y}$ b)
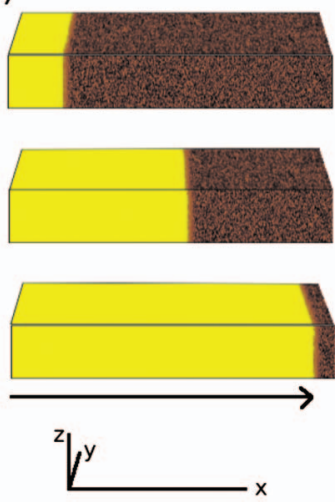

FIG. 3. Evolution of a front in a heterogeneous medium with weak links $\left(D^{(2)}=0.05, \phi=0.3\right)$ for 2D (a) and 3D (b). The three panels show the front for $t=2.25, t=6$, and $t=9.75$ t.u.. Fronts (bright regions) evolve from left to right, in the direction of the arrows. Different levels of dark color are plotted representing the heterogeneities. Model parameters: $k^{(1)}=50, D^{(1)}=1$, and $a=0.1$. Numerical parameters: $\Delta t=0.002$ time units (t.u.) and $\Delta x=0.1$ space units (s.u.). Size of the system: $10 \times 30$ s.u. $^{2}$ (a) and $5 \times 5 \times 30$ s.u. $^{3}$ (b). Periodic boundary conditions are applied in the directions $y$ and $z$.

rise to site percolation in the limit $D^{(2)}=0$.

(5) Nonreactive defects: Finally, the defects can also be nonreactive $\left(k^{(2)}=0\right)$, see Fig. 2(f). [In the limit $D^{(2)}$ $=0$, systems with both types of defects, the nonreactive $\left(k^{(2)}=0\right)$ and reactive $\left(k^{(2)}=k^{(1)}\right)$ ones, are equivalent.]

We carried out numerical simulations for all the cases listed above. We generated a front on one side of the system, which then propagated through the heterogeneous medium. Figure 3 shows two examples of front propagation in 2D and 3D with the same type (weak links) and fraction of heterogeneities. For both types of front, differences in the front velocity are observed for the same conditions. The front roughness is high and front propagation is irregular, especially for small $D^{(2)}$ and intermediate fractions of heterogeneities $\phi$ close to the percolation threshold $\phi^{*}$. The features of the patterns are qualitatively similar for the different cases of heterogeneities considered here.

Below, we present results obtained in 3D systems mainly, but similar conclusions can be drawn from an analysis of 2D dynamics (see Ref. 23).

\section{COMPARISON BETWEEN SIMULATION AND THEORY}

When the diffusion coefficients of both phases, $D^{(1)}$ and $D^{(2)}$, are large enough, i.e., both diffusion lengths are larger than the characteristic length of the heterogeneity, the effective medium theory described above can be compared to the numerical results of simulations of the cases described in the previous section.

In our simulations, we measured the front velocity in 3D for different values of the fraction $(\phi)$, diffusion coefficient $\left(D^{(2)}\right)$, and rate coefficient $\left(k^{(2)}\right)$ and compared these results with the analytical predictions of the effective medium theory. In the next sections we consider the case of $D^{(2)}>0$. The special case $D^{(2)}=0$ is outside the domain of the appli- 

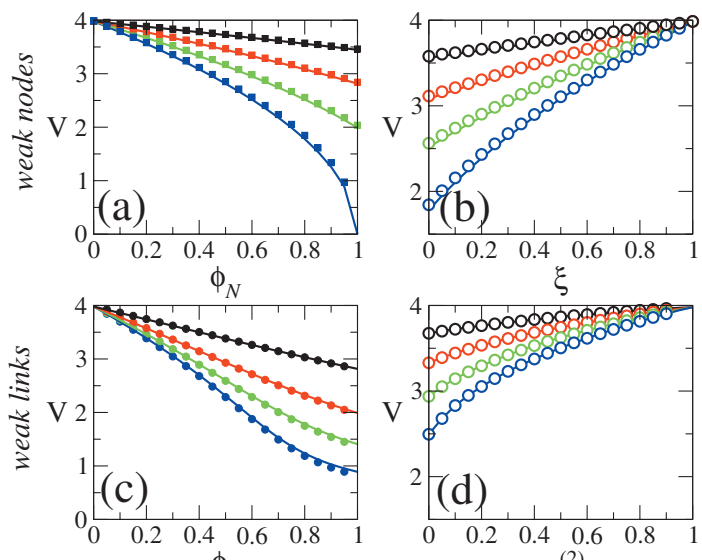

$\phi_{L}$
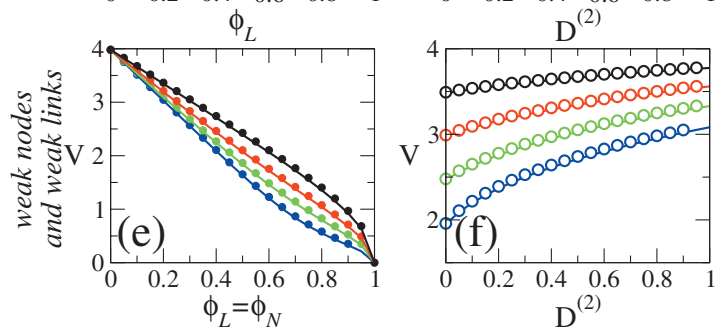

FIG. 4. Front velocity in 3D heterogeneous media with weak nodes [(a) and (b)], weak links [(c) and (d)], and weak links and nodes [(e) and (f)]. Left column shows the dependence of the velocity on the fraction of obstacles $[(\mathrm{a}),(\mathrm{c})$, and (e)] and the right column shows the dependence on the reactivity parameter $\xi$ (b) and on the diffusion (d) and (f). The various curves show different values of the reactivity (a), from top to bottom: $\xi=0.75, \xi$ $=0.5, \xi=0.25$ and $\xi=0$ (solid squares); diffusion [(c) and (e)], from top to bottom: $D^{(2)}=0.5, D^{(2)}=0.25, D^{(2)}=0.125$, and $D^{(2)}=0.05$ (solid circles); and fraction [(b), (d), and (f)], from top to bottom: $\phi=0.1, \phi=0.2, \phi=0.3$ and $\phi=0.4$ (open circles). Points are the results of an average over three realizations and solid lines are the theoretical predictions; parameters as in Fig. 3(b).

cability of the theory and, although some results can be obtained, this limit is not discussed here. For a discussion of this limit, see Ref. 23.

\section{A. Weak nodes}

We consider systems with a fraction $\phi_{N}$ of weak nodes with a reduced rate constant $k^{(2)}=\xi k^{(1)}$, where $0<\xi<1$. For small fractions of weak nodes the front velocity decreases, while for large fractions $\left(\phi_{N} \approx 1\right)$ and weak reactivities $(\xi$ $\approx 0$ ) the front stops. Using the expression obtained in Eq. (15) we obtain an effective reaction rate $k^{e}=(1-(1$ $\left.-\xi) \phi_{N}\right) k^{(1)}$ and introduce this expression into formula Eq. (30), keeping $D^{e}=D^{(1)}$. The dependence predicted by the effective medium theory correctly reproduces the simulation velocities for the entire range of $\phi_{N}$ and $\xi$ parameters, as can be verified by an examination of Figs. 4(a) and 4(b).

\section{B. Weak links}

Assuming spatially homogeneous reaction conditions, we suppose that some of the links $\left(L_{i j}\right)$ allow only a reduced flux between the nodes. The fraction of weak links with reduced diffusion coefficients $\left(D^{(2)}\right)$ is $\phi_{L}$ and its distribution is completely random. The front velocity decreases with increasing $\phi_{L}$. Since the reaction rate coefficients of the sites remains homogeneous $\left(k^{(1)}\right)$, we can calculate the front velocity by using Eq. (30) and substituting the effective diffu- sion coefficient obtained in Eq. (31), together with a constant reactivity $k^{e}=k^{(1)}$. The comparison between this analytical expression and the simulation results are shown in Figs. 4(c) and 4(d). The analytical curves reproduce the simulation results well for all of the $\phi_{L}$ and $D^{(2)}$ parameter values without the introduction of any fitting parameter.

\section{Weak links with weak nodes}

In order to study the effects of both heterogeneous reaction and diffusion terms, we consider the combination of weak links and weak nodes. We suppose that the fraction of weak links $\left(\phi_{L}\right)$ and weak nodes $\left(\phi_{N}\right)$ is the same $\phi_{L}=\phi_{N}$, but their positions are uncorrelated. The simulation results can be reproduced by the introduction of an effective diffusion coefficient $D^{e}\left(\phi_{L}\right)$ according to Eq. (31) and an effective reaction rate $k^{e}\left(\phi_{N}\right)$ using Eq. (32) into the formula for the velocity, Eq. (30). The comparison between theory and simulation is shown in Figs. 4(e) and 4(f).

\section{Defects}

The system with weak links studied in the previous sections yields bond percolation in the limit $D^{(2)}=0$. Now, we consider defects, which, in the same limit of $D^{(2)}=0$, yield site percolation.

We have performed numerical simulations of the reaction-diffusion system with a fraction $\phi_{D}$ of defects. In order to interpret the results of these simulations, one has to take into account the fact that the transport phenomena occur through the grid of links $\left(L_{i j}\right)$ and the defects are defined at the nodes of the grid $\left(N_{i}\right)$. To find an analytic expression for this case we have to transform the probability $\phi_{D}$ that a node does not diffuse to a different probability $\phi_{D L}$ for the links connected to such node. If $\phi_{D}$ is the fraction of defects and $1-\phi_{D}$ of that of normal nodes, the fraction of a normal link corresponds to $\left(1-\phi_{D}\right)^{2}$ and the fraction of a weak link associated to a defect is $\phi_{D L}=1-\left(1-\phi_{D}\right)^{2}$. Therefore, we define the probability of a weak link associated to a defect as $\phi_{D L}=2 \phi_{D}-\phi_{D}^{2}$, and we substitute the probability $\phi_{D}$ by the new probability $\phi_{D L}$ in Eq. (31) giving rise to $D^{e}\left(\phi_{D L}\right)$. The resulting expressions are plotted in Figs. 5(a) and 5(b) for different values of $D^{(2)}$ and the fraction of defects $\phi_{D}$. Note that the horizontal axis in Fig. 5 is $\phi_{D}$ and not $\phi_{D L}$ because $\phi_{D}$ is the control parameter in the numerical simulations. This approach reproduces the numerical results for large values of $D^{(2)}$, as expected, but the disagreement between the theory and the simulations become evident at small values of $D^{(2)}$.

\section{E. Nonreactive defects}

In the previous case, we focused on the effect of defects in the transport properties of reaction-diffusion systems. It seems reasonable to couple defects to nonreactive nodes giving rise to nonreactive defects. However, both types of disruptions are implemented at different grid levels. First, the defects affect the transport in the grid of links $\left(L_{i j}\right)$ and, therefore, we have to transform the fraction of defects $\phi_{D}$ to the fractions of defect links $\phi_{D L}$ in the expression for the effective diffusion coefficient Eq. (31), $D^{e}\left(\phi_{D L}\right)$. Second, the 

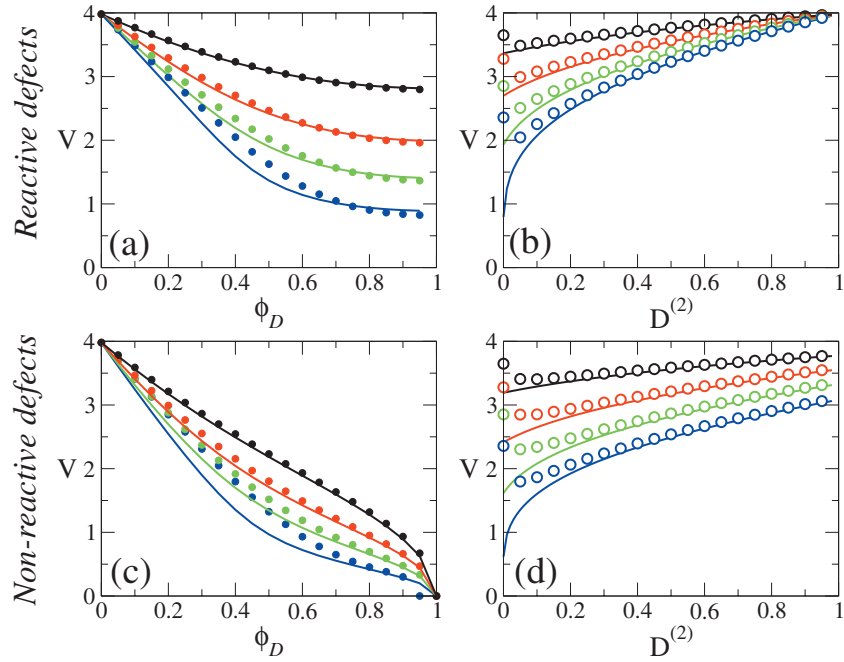

FIG. 5. Front velocity in 3D heterogeneous media with reactive [(a) and (b)] and nonreactive $[(\mathrm{c})$ and (d)] defects. Left column shows the dependence of the velocity on the fraction $\phi_{D}[(\mathrm{a})$ and (c) $]$ and the right column on the diffusion coefficient $D^{(2)}[(\mathrm{b})$ and (d)]. The plots show different values of the diffusion coefficient in the defects [(a) and (c)], from top to bottom: $D^{(2)}$ $=0.5, D^{(2)}=0.25, D^{(2)}=0.125$, and $D^{(2)}=0.05$ (solid circles); and fraction of the defects [(b), (d), and (f)], from top to bottom: $\phi=0.1, \phi=0.2, \phi=0.3$ and $\phi=0.4$ (open circles). Points are the average over three realizations and solid lines are the theoretical predictions; parameters as in Fig. 3(b).

nonreactivity affects the reaction in the grid of nodes $\left(N_{i}\right)$ and we can still use the fraction $\phi_{D}$ in the expression for the effective reaction rate Eq. (32), $k^{e}\left(\phi_{D}\right)$.

Figures 5(c) and 5(d) show the comparison between the simulations and the modified effective theory. As in the previous case there is a good agreement for large values of the diffusion coefficient in the defects, but substantial differences are observed for small diffusion coefficients.

\section{FURTHER APPLICATIONS OF EFFECTIVE MEDIUM THEORY AND ITS LIMITS}

This section is devoted to the discussion of possible extensions of the previous results. The two cases presented here are examples of heterogeneous reaction-diffusion media with some interesting features but an exhaustive study of these systems is not made. First, we compare the results of the effective medium theory, which was derived assuming a random distribution of heterogeneities with simulations for regular patterns of heterogeneities. Second, we study the effects of spatio-temporal heterogeneities.

\section{A. Comparison with regular inhomogeneities}

The existence of a random distribution of heterogeneities was one of the main assumptions of the theory discussed above. As a result of this assumption large distance correlations are precluded. Here, we compare the results obtained from the effective medium theory with simulations of reaction-diffusion systems with a regular distribution of heterogeneities where long range correlations exist. We introduce different types of periodic structures having constant fractions of heterogeneities $(\phi)$, and study the effect of different values of the diffusion coefficient in the particular case of nonreactive defects.

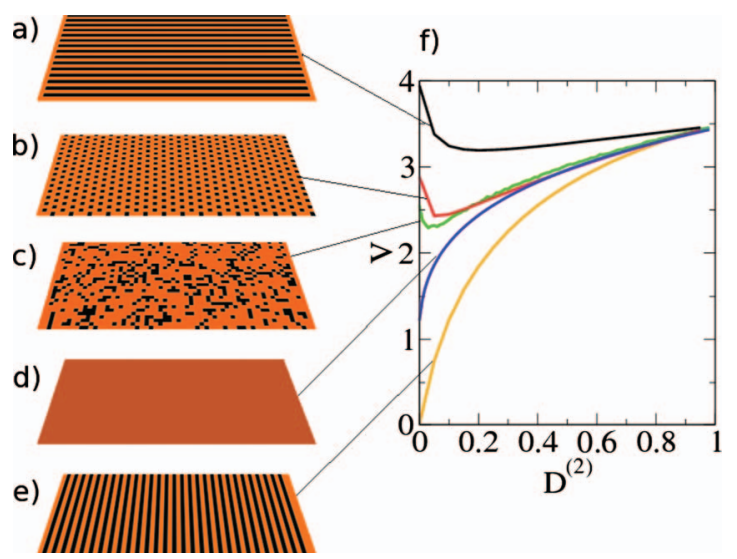

FIG. 6. Front velocity in 2D heterogeneous media with nonreactive defects for different distributions of heterogeneities. The fraction of defects is kept constant at $\phi_{D}=0.25$. The panels correspond to (a) linear heterogeneities oriented parallel to the direction of the front (horizontal bands), (b) regular grid of individual heterogeneities (hexagonal), (c) random heterogeneities, (d) theoretical prediction with the effective medium theory, and (e) linear heterogeneities oriented perpendicular to the direction of the front (vertical bands). Parameters as in Fig. 3(a).

The regular patterns considered in Fig. 6 are horizontal and vertical bands as well as a hexagonal grid of heterogeneities. In the limit where $D^{(2)}=D^{(1)}$, all distributions give rise to similar velocities, although they are not equivalent since they have different spatial distributions of nonreactive nodes.

As the control parameter $D^{(2)}$ decreases the two bandtype distributions, Figs. 6(a) and 6(e) quickly diverge from the theoretical prediction and have quite different values for $D^{(2)}=0$. In this limit the distribution with horizontal bands [Fig. 6(a)] gives rise to an array of independent homogeneous one dimensional systems where the front propagates with the maximum velocity. However, the distribution with perpendicular bands [Fig. 6(e)] for $D^{(2)}=0$ causes the front propagation to stop because the front cannot even cross the first band (equivalent to 1D system).

The hexagonal [Fig. 6(b)] and random [Fig. 6(c)] distributions have similar properties and the effective medium theory reproduces the values of the front velocity for intermediate and large values of $D^{(2)}$. The numerical simulations show, however, an increase in the velocity for small values of $D^{(2)}$. Such a velocity increase can be attributed to effects similar to those observed for horizontal bands where the low value of the diffusion in the heterogeneities prevents any flux of material into them. As a result the front can propagate faster than for larger values of $D^{(2)}$.

\section{B. Spatiotemporal disorder}

We have so far supposed that the heterogeneities are static. Here, we introduce disorder with a temporal dynamics by changing the realization of the random distribution of heterogeneities after a time $\tau$. We focus here on the random variation of the diffusion coefficients; interactions between nonlinear reaction rates and random forces have been extensively studied in reaction-diffusion media. ${ }^{31}$

In Fig. 7 we show the results of such dynamics for weak links. For this case, we find that the front velocity depends 
a)
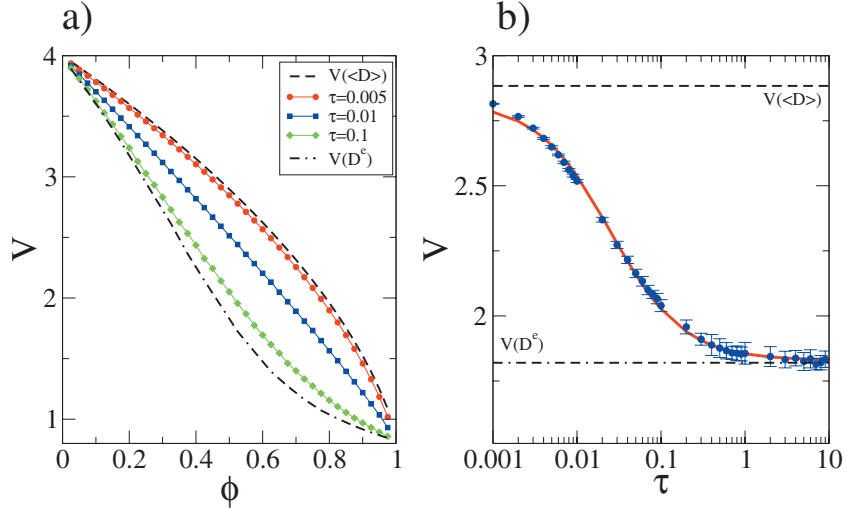

FIG. 7. Dependence of the front velocity on the fraction of heterogeneities $\left(\phi_{L}\right)$ for different correlation times of the dynamic disorder (a), and on the correlation time $\tau$ for $\phi_{L}=0.5$ (b). Dashed and dot-dashed lines correspond to the theoretical values of the velocity for an arithmetic average of the diffusion coefficient $\mathrm{V}(\langle D\rangle)$ and for the effective diffusion coefficient $\mathrm{V}\left(D^{e}\right)$, respectively. Points correspond to an average over ten realizations. Parameters as in Fig. 3(a).

on the relation between the correlation time $\tau$ and the diffusion time $\tau_{D^{e}}=\ell^{2} / D^{e}$. As expected, for large values of $\tau$ the dependence of the velocity on the fraction of heterogeneities is the same as that for the static case (limit $\tau \rightarrow \infty$ ). As the correlation time decreases, the fronts travel faster. For correlation times small compared to the diffusion time $\left(\tau \ll \tau_{D^{e}}\right)$, the velocity saturates to a value that can be calculated from the arithmetic mean of the diffusion coefficients,

$$
\langle D\rangle=D^{(1)}\left(1-\phi_{L}\right)+D^{(2)} \phi_{L},
$$

by the replacement of $D^{e}$ by $\langle D\rangle$ in Eq. (30). Panel b in Fig. 7 shows the dependence of the velocity on the characteristic time, for constant fraction of disorder.

For $\tau \ll \tau_{D^{e}}$ the realization of the disorder changes repeatedly during the time for diffusion of the species over the characteristic size of the heterogeneity $(\ell)$. The selfcontribution in Eq. (17) is zero in this limit. This condition is equivalent to assuming that $g_{d}=0$, and the introduction of such a value in Eq. (26) gives rise to Eq. (33).

\section{DISCUSSION}

In the effective medium theory for reaction-diffusion systems considered here heterogeneities in the reaction rate give rise to an effective reaction rate, which is the arithmetic average of the two rates [Eq. (15)], while heterogeneities in diffusion yield an effective diffusion coefficient, which can be calculated from Eq. (27) and coincides with the formula of Bruggeman. ${ }^{26}$ Our comparisons of the theory with simulations show that the effective medium theory can be applied directly to the case of weak links, but a modified theory, based on a corrected fraction of heterogeneities, is needed for the description of defects. We found good agreement between the simulation results and the effective medium theory for all cases with large diffusion coefficients in the heterogeneities $\left(D^{(2)}\right)$. Although the results presented here were obtained for a simple model, many of the conclusions are applicable to more complex biological, physical, and chemical reaction-diffusion systems.
Pulses in excitable media have features that are similar to those of fronts in bistable media. The main difference is that excitable systems relax back to the initial rest state after some recovery time. If the size of the heterogeneities is smaller than the characteristic size of a pulse, one can apply the results of this study to heterogeneous excitable media. Cardiac tissue is an example of a heterogeneous excitable medium. There are several numerical studies of pulse propagation in heterogeneous cardiac tissue ${ }^{14,12,13}$ and there are also some investigations of the construction of homogenization methods for cardiac tissue. For instance, periodic changes in the diffusion coefficient can be averaged and an expression for the velocity can be obtained that depends on the particular structure of the reaction-diffusion model. ${ }^{20}$ Our results are independent of the details of the model and assume a random distribution of heterogeneities. Cell cultures are highly heterogeneous excitable systems. They are composed of a large number of cells, which are interconnected in a complex way. For such cell cultures a linear dependence of the velocity on the density of electric sinks has been predicted. ${ }^{15}$ The application of our results to this case is more complicated because the characteristic sizes of the pulses and the heterogeneities are comparable.

The results presented here should prove useful in study of several types of chemical pattern formation process, for example, the patterns in the heterogeneous BelousovZhabotinsky reaction, where some of the reactants diffuse freely in the system while other reactants are confined to reverse micelles in microemulsions or porous catalytic particles. One can consider the compartments as heterogeneities with different reaction properties and very slow diffusion. Further studies of more complex reaction-diffusion systems can be carried out using the framework developed here.

\section{ACKNOWLEDGMENTS}

We acknowledge financial support from the German Science Foundation (DFG) within the framework of SFB 555 "Complex Nonlinear Processes." Research of R.K. was supported in part by NSERC.

${ }^{1}$ R. C. Desai and R. Kapral, Dynamics of Self-Organized and SelfAssembled Structures (Cambridge University, Cambridge, England, 2009).

${ }^{2}$ M. C. Cross and P. C. Hohenberg, Rev. Mod. Phys. 65, 851 (1993).

${ }^{3}$ Chemical Waves and Patterns, edited by R. Kapral and K. Showalter (Kluwer, Dordrecht, 1994).

${ }^{4}$ J. P. Keener and J. Sneyd, Mathematical Physiology (Springer, New York, 1998).

${ }^{5}$ I. Sendiña-Nadal, A. P. Muñuzuri, D. Vives, V. Pérez-Muñuzuri, J. Casademunt, L. Ramírez-Piscina, J. M. Sancho, and F. Sagués, Phys. Rev. Lett. 80, 5437 (1998).

${ }^{6}$ O. Steinbock, P. Kettunen, and K. Showalter, Science 269, 1857 (1995).

${ }^{7}$ M. Bär, A. K. Bangia, I. G. Kevrekidis, G. Haas, H.-H. Rotermund, and G. Ertl, J. Phys. Chem. 100, 19106 (1996).

${ }^{8}$ V. K. Vanag and I. R. Epstein, Science 294, 835 (2001).

${ }^{9}$ A. F. Taylor, M. R. Tinsley, F. Wang, Z. Huang, and K. Showalter, Science 323, 614 (2009).

${ }^{10}$ M. R. Tinsley, A. F. Taylor, Z. Huang, and K. Showalter, Phys. Rev. Lett. 102, 158301 (2009).

${ }^{11}$ V. K. Vanag and I. R. Epstein, Phys. Rev. Lett. 87, 228301 (2001).

${ }^{12}$ K. H. W. J. Ten Tusscher and A. V. Panfilov, Phys. Rev. E 68, 062902 (2003).

${ }^{13}$ K. H. W. J. Ten Tusscher and A. V. Panfilov, Europace 9, vi38 (2007). 
${ }^{14}$ A. V. Panfilov, Phys. Rev. Lett. 88, 118101 (2002).

${ }^{15}$ B. E. Steinberg, L. Glass, A. Shrier, and G. Bub, Philos. Trans. R. Soc. London, Ser. A 364, 1299 (2006).

${ }^{16}$ G. Bub, A. Shrier, and L. Glass, Phys. Rev. Lett. 88, 058101 (2002).

${ }^{17}$ R. Thul and M. Falcke, Phys. Rev. Lett. 93, 188103 (2004).

${ }^{18}$ S. Rüdiger, J. Shuai, W. Huisinga, C. Nagaiah, G. Warnecke, I. Parker, and M. Falcke, Biophys. J. 93, 1847 (2007).

${ }^{19}$ E. Sykova and C. Nicholson, Physiol. Rev. 88, 1277 (2008).

${ }^{20}$ J. P. Keener, Physica D 136, 1 (2000).

${ }^{21}$ J. Xin, SIAM Rev. 42, 161 (2000).

${ }^{22}$ O. Runborg, C. Theodoropoulos, and I. G. Kevrekidis, Nonlinearity 15, 491 (2002).
${ }^{23}$ S. Alonso, R. Kapral, and M. Bär, Phys. Rev. Lett. 102, 238302 (2009).

${ }^{24}$ D. Bedeaux and R. Kapral, J. Chem. Phys. 79, 1783 (1983).

${ }^{25}$ We have assumed that the diffusion matrix is diagonal.

${ }^{26}$ D. A. G. Bruggeman, Ann. Phys. 416, 636 (1935).

${ }^{27}$ S. Torquato, Random Heterogeneous Materials (Springer, New York, 2002).

${ }^{28}$ S. Kirkpatrick, Phys. Rev. Lett. 27, 1722 (1971).

${ }^{29}$ S. Kirkpatrick, Rev. Mod. Phys. 45, 574 (1973).

${ }^{30}$ V. K. Vanag and I. R. Epstein, Phys. Chem. Chem. Phys. 11, 897 (2009).

${ }^{31}$ F. Sagués, J. M. Sancho, and J. García-Ojalvo, Rev. Mod. Phys. 79, 829 (2007). 